\title{
Guider les étudiants universitaires vers l'autorégulation dans leur apprentissage en ligne
}

\section{Daouas Thouraya}

Institut des Hautes Études Commerciales, Carthage-Présidence, TUNISIE

Thouraya.Daouas@ihec.rnu.tn

\section{Compte rendu de pratique}

\section{Résumé}

Dans cette étude, des étudiants universitaires ont eu un premier contact avec un cours en ligne d'algorithmique de base mis à leur disposition en parallèle avec leur cours classique en présentiel. Ce cours en ligne est considéré comme une deuxième source d'apprentissage afin de leur permettre de se détacher relativement de leur cours en classe et d'avoir un peu plus d'autonomie. En même temps, nous avons distribué aux étudiants un guide pour l'apprentissage autorégulé pour les aider à retrouver les stratégies spécifiques dans un contexte déterminé d'apprentissage. Nous présentons dans cet article les résultats, que nous avons obtenus par un questionnaire distribué aux étudiants à la suite de leur test de fin du cours.

\section{Abstract}

In this research, university students came into contact for the first time with an online course on basic algorithms, placed at their disposal in parallel with the regular course. In this context, the online course was considered a secondary educational source, one that enabled them to be relatively detached from their classroom course and act somewhat more autonomously. Students also received a self-regulated learning guide to help them pinpoint specific strategies in a given learning context. This paper presents the results obtained from the questionnaire distributed to the students as well as from their end-ofcourse test results.

(CAuteur(s). Cette œuvre, disponible à http://www.ritpu.org/IMG/pdf/ritpu0401_thouraya.pdf, est mise à disposition selon les termes de la licence Creative Commons Attribution - Pas de Modification 2.5 Canada : http://creativecommons.org/licences/by-nd/2.5/ca/deed.fr 


\section{Introduction}

Dans notre ère, la technologie se développe rapidement et à tous les niveaux de la vie de l'étudiant universitaire. Cependant, il est assez évident que ce dernier se retrouve en situation de relative passivité qui laisse peu de place à l'initiative (Naymark, 1999, cité par Abdelli, 2003). L'étudiant a un rôle de récepteur lors de la majorité des cours. Il doit, globalement, acquérir le cours de l'enseignant et le réviser pour passer l'examen. De cette façon, l'apprentissage s'arrête généralement à la sortie de l'université. Cependant, une des fonctions majeures de l'éducation est le développement des compétences à l'apprentissage chez l'étudiant tout au long de sa vie active. Ainsi, après l'obtention de son diplôme universitaire, il doit acquérir d'autres compétences importantes d'une manière informelle (Zimmerman, 2002). Les chercheurs ont montré clairement que les étudiants qui sont autorégulés dans leur apprentissage ont les capacités d'autodéterminer des stratégies pour apprendre à accomplir leurs tâches d'une manière de plus en plus satisfaisante (Deci et Ryan, 2000; Pintrich, 2000).

L'autorégulation n'est pas une caractéristique innée chez l'individu. C'est un processus d'apprentissage qui s'acquiert et qui doit être nourri et renforcé continuellement. C'est pour cette raison que plusieurs ont tenté de trouver des méthodes d'enseignement ou des modèles d'autorégulation (Lebow, 1993; Lucas, 1990).

Par ailleurs, il y a un besoin croissant d'enseignement continu qui dépasse les barrières des institutions éducatives, mais tandis que la demande grandit, le défi devient de plus en plus grand pour le secteur public (Roadmap to communicating knowledge essential for the indusTrial environment [ROCKET] consortium, 2003). De plus, la psychologie éducationnelle ajoute que la combinaison de plusieurs canaux d'apprentissage accentue ce défi. Le eLearning offre, dans ce contexte, des moyens inédits pour mettre en œuvre ce principe (Balancier, Georges, Jacobs, Martin et Poumay, 2006). En effet, le eLearning permet de cumuler les avantages de la person- nalisation et de la démultiplication. Cependant, il représente un changement important dans les habitudes de travail des apprenants et des enseignants.

\section{Objectif et contexte}

Les étudiants de l'Institut des Hautes Études Commerciales de Carthage obtiennent leur diplôme de maîtrise à la suite de quatre années d'études. Cette maîtrise est en gestion avec une option déterminée telle que Finance, Marketing, Commerce international, etc. Au cours de leur formation en 2e année, ces étudiants ont un cours d'informatique de base qui les initie à l'algorithmique. Les cours donnés aux étudiants pendant les quatre années sont des cours magistraux traditionnels de type présentiel. Chaque cours dure un semestre et se termine par un examen à la fin du semestre.

Dans le but de mesurer le degré de prédisposition de ces étudiants à devenir plus autorégulés et plus autonomes dans leur apprentissage, nous leur avons fourni le Guide d'Apprentissage pour l'Apprenant Autorégulé (G3A), que nous avons élaboré. De plus, et afin de profiter des avantages du eLearning, nous leur avons donné accès à un cours d'algorithmique en ligne. Ce cours a deux rôles : le premier est de leur permettre de pratiquer un apprentissage en ligne en plus de leur cours classique en présentiel et le deuxième est d'appuyer leur autonomie dans l'apprentissage.

À la suite de l'utilisation du guide et du cours en ligne, nous avons pu mesurer aussi le degré de motivation des étudiants vis-à-vis du cours en ligne et du guide élaboré, ce qui nous a permis d'envisager de possibles perspectives pour ce travail.

Ce texte parle d'une expérimentation effectuée dans le cadre d'un cours d'algorithmique enseigné à des étudiants universitaires. Après la définition de l'autorégulation, nous explicitons le Guide d'Apprentissage pour l'Apprenant Autorégulé, que nous avons élaboré, ainsi que le site en ligne. Nous décrivons par la suite l'échantillonnage et la procédure suivie. Pour 
finir, nous présentons les résultats obtenus et les conclusions que nous avons pu tirer de cette expérimentation.

\section{Apprentissage autorégulé}

L'apprentissage autorégulé est défini par Pintrich (2000) comme un processus actif et constructif par lequel les apprenants fixent leurs objectifs d'apprentissage et exercent le suivi et le contrôle de leurs connaissances, motivations et comportements. Ils sont guidés dans ce processus par leurs objectifs et leurs environnements d'apprentissage. De plus, l'apprenant autorégulé concentre ses efforts sur la maîtrise des tâches académiques, telles que lire ses notes, préparer ses cours, gérer son temps et respecter les délais, et sur l'amélioration des compétences et de la compréhension. Il n'est pas concerné par la compétition avec les camarades de classe et l'évaluation de soi par rapport aux autres (Ames, 1992; Pintrich et Schunk, 1996). L'autorégulation consiste à approcher une tâche d'apprentissage avec confiance, assiduité et ingéniosité. L'apprenant est alors conscient de son degré de maîtrise et de connaissance d'un objet étudié. Il recherche l'information dont il a besoin et procède à son assimilation. Il est capable de trouver le moyen de réussir lorsqu'il fait face à des obstacles tels que de mauvaises conditions d'étude, des professeurs qui n'arrivent pas à faire passer le message ou l'indisponibilité de livres sur la matière, etc. (Wilson, 1997). L'autorégulation présume que l'apprenant qui est actif et qui contrôle ses apprentissages est plus performant que les autres.

Afin de mesurer à quel degré les étudiants sont prédisposés à devenir plus autorégulés, nous avons élaboré le Guide d'Apprentissage pour l'Apprenant Autorégulé, que nous détaillons dans la section suivante.

\section{Guide d'Apprentissage pour l'Apprenant Autorégulé (G3A)}

Les chercheurs ont affirmé que l'utilisation de stratégies d'apprentissage effectives joue un rôle important dans le développement de la motivation des étudiants (McCor- mick et Pressley, 1997). Les étudiants autorégulés appliquent différentes stratégies d'apprentissage appropriées à des tâches spécifiques (Lapan, Cardash et Turner, 2002). Ces stratégies doivent être connectées et liées, le plus possible, à des exemples du monde réel pouvant aider les étudiants à intégrer leur apprentissage avec un futur possible et réalisable.

Lors de l'élaboration du guide G3A, notre but était d'orienter et d'aider les étudiants à s'autoréguler dans leur apprentissage. Or, ce qui convient à un étudiant ne convient pas nécessairement à un autre et ce qui convient à un étudiant à un moment donné ne convient pas nécessairement à ce même étudiant à un autre moment. Nous avons donc conçu ce guide sous forme de directives et de propositions de stratégies pouvant aider les étudiants à mieux maîtriser les tâches académiques et à améliorer leurs compétences et leur compréhension. Chaque étudiant pourrait adopter les stratégies qui lui conviennent et même s'inspirer de ces stratégies pour en trouver de meilleures.

Le guide est composé de six rubriques principales, à savoir Fixation d'objectifs, Planification et gestion du temps, Stratégies d'apprentissage, Autocontrôle, Stratégies pour le passage du test et Autoréflexion.

\section{Fixation d'objectifs}

Le fait de se fixer des objectifs permet à l'apprenant de délimiter le champ des options et celui des outils à utiliser. Par conséquent, l'étudiant peut faire des économies considérables de temps pour démarrer et accomplir son apprentissage. En outre, il peut juger de son état d'avancement et du fait qu'il a atteint son objectif ou non parce qu'il sait exactement où il veut aboutir (Zimmerman, Bonner et Kovach, 1996).

Il est nécessaire de se fixer des objectifs appropriés et réalisables. Ces derniers doivent provenir de l'individu lui-même et non d'une autre personne. En plus, les objectifs doivent porter une orientation d'apprentissage et de maîtrise d'un aspect et non de performance (Anderman et Maehr, 1994). En effet, les étudiants qui se fixent l'objectif d'acquérir des 
connaissances dans une matière sont plus motivés et plus aptes à surmonter leurs frustrations que ceux qui se fixent l'objectif d'être les premiers de la classe. Les objectifs, ainsi fixés, présentent un défi plus efficace pour l'apprenant et affectent positivement l'autorégulation de l'étudiant (Paris et Winograd, 1997). Il existe deux types d'objectifs, à savoir des objectifs à long terme et des objectifs à court terme. L'accomplissement des objectifs à long terme nécessite des mois, voire un semestre (ex. : achever les chapitres du cours), alors que celui des objectifs à court terme nécessite quelques jours ou semaines (ex. : accorder plus de temps aux devoirs à la maison).

\section{Planification et gestion du temps}

Il est essentiel que l'apprenant gère son temps et ses ressources par une planification et un contrôle effectifs afin d'établir ses priorités, surmonter la frustration et persister jusqu'à l'accomplissement de la tâche. Cette planification fait gagner du temps et de l'énergie aux apprenants, mais elle suppose une capacité de distinguer entre ce qui est important et ce qui l'est moins (Paris et Winograd, 1997). Pratiquement, l'étudiant devra faire un plan à partir de ses objectifs à court terme, fixés au préalable, pour aboutir à une liste d'étapes à réaliser. Ces étapes seront accompagnées d'une liste de problèmes potentiels et de possibilités pour les résoudre.

\section{Stratégies d'apprentissage}

Les stratégies d'apprentissage peuvent être définies comme des opérations qu'un individu, engagé dans un processus de formation, effectue dans le but de favoriser l'acquisition de savoirs, d'habiletés ou d'attitudes (Hrimech, 2000; Ruph et Hrimech, 2001; Wade, Trathern et Schaw, 1990; Weinstein, 1988). Ces stratégies se réfèrent à toute activité technique ou procédure utilisée par les apprenants pour améliorer leur compréhension et leur performance lors d'une tâche d'apprentissage (Alexander, Graham et Harris, 1998; Mayer, 1996). Cependant, il n'existe pas de stratégies efficaces dans toutes les situations, mais dans un contexte donné. De plus, l'efficacité d'une stratégie dépend de son adaptabilité à l'individu qui l'utilise. Nous avons classifié les stratégies selon différents types (McCormick et Pressley, 1997) :
- stratégies d'organisation pour mieux s'organiser dans son travail d'apprentissage;

- stratégies sociales permettant d'apprendre des personnes de son entourage et d'exploiter leurs compétences;

- stratégies de l'apprentissage actif évitant à l'étudiant de s'évader ou de se relâcher au cours de son apprentissage; et

- stratégies cognitives permettant à l'étudiant de mieux assimiler et mémoriser à long terme les informations révisées.

\section{Autocontrôle}

$C^{\prime}$ est une remise en cause qui traduit un engagement personnel de l'étudiant à la performance. L'étudiant devra évaluer l'efficacité de sa stratégie d'apprentissage. Une stratégie jugée efficace devra être retenue comme stratégie à appliquer dans ce contexte d'apprentissage précis. En $d^{\prime}$ autres termes, ce serait une erreur d'utiliser une stratégie, réussie dans un cadre particulier, dans toutes les situations d'apprentissage. Par contre, il faudra identifier un ensemble de stratégies efficaces, chacune pour une situation spécifique, afin de les utiliser si une situation semblable se présente (Svinicki, 2004).

L'autocontrôle se fait d'une manière continue au niveau de chaque étape de l'apprentissage. Ceci se fait dans le respect de la planification et de l'atteinte des objectifs à court terme. Ainsi, atteindre les objectifs à court terme induit l'atteinte de l'objectif à long terme.

\section{Stratégies pour le passage du test}

Il est important de signaler à ce niveau qu'une bonne préparation pour un test suppose au préalable une bonne gestion du temps, de bonnes prises de notes et une révision régulière des concepts étudiés. Une révision se traduit par une revue hebdomadaire, voire journalière du cours. Une revue du test est aussi nécessaire pour distinguer entre les concepts maîtrisés et ceux qui le sont moins pour les mémoriser définitivement.

\section{Autoréflexion}

L'autojugement et l'autoréaction dans l'apprentissage sont la clé de l'autorégulation. L'étudiant devra pouvoir 
évaluer le degré d'atteinte de ses objectifs d'apprentissage préfixés et devra savoir réagir à l'échec (Svinicki, 2004). En effet, l'échec constitue un grand obstacle à l'apprentissage autorégulé. Les étudiants ont alors une tendance à douter de leurs capacités personnelles et se découragent avant d'atteindre leurs objectifs. Cependant, ils oublient de considérer le fait que surmonter l'échec est plus important que l'échec en soi (Paris et Winograd, 1997).

\section{Cours en ligne}

Parmi les unités de cours enseignées actuellement dans nos universités, l'algorithmique fait partie des cours indispensables pour la formation des étudiants, car elle représente un premier contact avec l'informatique. L'intérêt d'un tel cours est de permettre aux futurs diplômés de mieux comprendre la façon de réaliser des programmes afin de pouvoir plus facilement utiliser l'ordinateur pour résoudre des problèmes scientifiques, techniques ou organisationnels. Étant donné que la majorité des cours se font en présentiel, nous avons voulu accompagner ce cours d'algorithmique d'un site en ligne nommé NF01, décrit dans Trigano (2006), accessible en ligne (http: / / www4.utc.fr / nf01/fc-frameset-accueil1.htm) et réalisé à l'Université de Technologie de Compiègne en France. L'objectif de ce site NF01 est, d'une part, de familiariser l'étudiant à l'utilisation de l'ordinateur, et d'autre part, de l'aider à commencer l'apprentissage de l'algorithmique et de la programmation. L'étudiant devra ainsi apprendre à trouver la solution d'un problème à l'aide d'une forme algorithmique et savoir programmer cette solution algorithmique en un langage structuré. En outre, l'utilisation de ce site permettra à l'étudiant d'avoir une source d'apprentissage autre que son enseignant, ce qui l'inciterait à réaliser ses propres tâches avec ses propres stratégies en vue de devenir au fur et à mesure plus autonome et plus autorégulé.

Le cours en ligne d'algorithmique NF01 se compose des liens suivants :

- Théorique : c'est un lien vers les chapitres du cours.

- Exercices : c'est un lien vers des exercices classiques cou- vrant tout le cours avec des énoncés et des corrections.

- Simulation : c'est un lien qui offre à l'étudiant des simulations des traitements des schémas de base de l'algorithmique, tels que l'affectation, les boucles, etc.

- QCM : c'est un lien vers des exercices sous forme de questions à choix multiples permettant à l'étudiant de vérifier ses connaissances.

- Jeu : c'est un lien vers un jeu pédagogique qui permet à l'étudiant de tester ses connaissances en algorithmique.

- Téléchargements : c'est un lien qui offre à l'étudiant le téléchargement des parties du cours ainsi que des exercices.

Nous avons voulu mesurer l'attitude et la motivation des étudiants envers chacune des parties présentées par les liens du site NF01 en vue de nous éclairer davantage sur le niveau d'autorégulation des étudiants, d'une part, et sur leurs préférences par rapport aux liens, d'autre part.

\section{Échantillonnage et procédure expérimentale}

Ce qu'il est important de mesurer, c'est le degré de présence des caractéristiques de l'autorégulation chez les étudiants. Pour cela, nous avons utilisé le cours NF01, d'une part, et le guide G3A, d'autre part. Nous avons choisi de travailler sur trois types d'échantillons que nous avons appelés $E_{0}, E_{\text {Net }}$ et $E_{\text {Net-G3A }}$. $E_{0}$ est un échantillon des étudiants suivant le cours de la manière traditionnelle, $\mathrm{E}_{\mathrm{Net}}$ est l'échantillon des étudiants suivant le même cours et auxquels nous avons donné l'adresse du site du cours en ligne NF01 à consulter en parallèle avec leurs cours et $\mathrm{E}_{\mathrm{Net-G3A}}$ est l'échantillon des étudiants suivant le cours et auxquels nous avons donné l'adresse du site du cours en ligne NF01 ainsi que le guide G3A.

Notre choix a été basé sur le fait que les étudiants des trois échantillons devaient suivre le même cours classique avec le même enseignant et devaient passer le même test pour que nous puissions comparer leurs notes de fin de cours. L'enseignant devait donner son cours sans tenir compte de nos échantillons et devait faire passer le même test à tous les étudiants. Nous nous sommes 
intéressé à trois types de comparaisons afin de mesurer

l'auto-estimation, l'estime de soi des étudiants ainsi que l'influence du cours NF01 et celle du guide G3A.

En plus, nous avons décidé de laisser $\mathrm{E}_{0}$ suivre le cours normalement sans l'informer de la procédure entreprise. Ceci nous éviterait les possibilités d'avoir des résultats biaisés au cas où quelques étudiants seraient tentés de consulter NF01 ou G3A. Pour cela, nous nous sommes basé sur la consultation des emplois du temps des étudiants pour choisir un échantillon $\mathrm{E}_{0}$ qui ne se croise pas avec les autres échantillons pendant les intercours. Pour $\mathrm{E}_{\mathrm{Net}}$ et $\mathrm{E}_{\mathrm{Net-G3A^{ \prime }}}$ le problème ne se posait pas, car ces deux échantillons avaient un accès au cours NF01. De plus, nous avons distribué à $\mathrm{E}_{\text {Net-G3A }}$ le guide G3A en papier et, par expérience, nous savions que nos étudiants ne prennent pas l'initiative de copier un document d'un autre groupe, se contentant toujours de ce que leur enseignant distribue en classe.

Enfin, comme les groupes d'étudiants du cours d'informatique sont constitués de 20 étudiants chacun au maximum, et pour satisfaire la contrainte d'avoir un même enseignant et celle de l'emploi du temps citées au préalable, nous sommes arrivé à obtenir trois échantillons de 20 étudiants chacun ayant des horaires de cours très éloignés.

Nous avons communiqué à $\mathrm{E}_{\mathrm{Net}^{\prime}}$ en présence de leur enseignant, l'adresse du site de NF01. De même, nous avons communiqué à $\mathrm{E}_{\mathrm{Net-G} \text { A }} \mathrm{l}^{\prime}$ adresse du site NF01 et nous avons distribué le guide G3A en papier à chaque étudiant de cet échantillon.

\section{Questionnaire}

À la fin du cours d'algorithmique, nous avons distribué un même questionnaire aux trois échantillons. Ce questionnaire est composé de trois parties distinctes. La motivation est souvent considérée comme le moteur de l'apprentissage (Organisation de coopération et développement économiques [OCDE], 2004, chap. 3). Par conséquent, nous avons consacré la première partie du questionnaire à la mesure de la motiva- tion des étudiants par rapport au cours NF01 et par rapport au guide G3A. Par ailleurs, puisque le type de motivation peut différer d'un étudiant à un autre, nous avons distingué trois formes de motivation : une motivation liée à l'intérêt qu'a l'étudiant pour la matière, telle que vouloir s'améliorer, une motivation liée à une certaine relation de pouvoir de la part du système des cours, telle que le fait que ce soit imposé, et une motivation liée à la personnalité de l'étudiant, telle que la curiosité. Cette distinction est importante dans le sens qu'elle pourra nuancer certains traits de la personnalité de l'étudiant et ainsi nous donner une idée de son autorégulation. Dans une deuxième partie, nous avons voulu mesurer l'attitude et la motivation des étudiants par rapport au cours NF01 en tant que cours en ligne et deuxième source d'apprentissage. Dans une troisième partie, le questionnaire vise à mesurer le degré d'autorégulation des étudiants et leur degré d'estime de soi en mesurant leur degré de confiance pour le passage du test. Pour finir, nous avons mesuré à travers le questionnaire le degré d'utilité du guide pour des cours autres que le cours d'algorithmique, ainsi que son influence possible sur la poursuite de l'apprentissage des étudiants après l'obtention de leur diplôme.

Par ailleurs, les étudiants des trois échantillons ont passé un même test préparé par leur enseignant. Nous avons choisi de distribuer le questionnaire à remplir par les étudiants avant le test parce que chaque étudiant devait indiquer un intervalle de sa note estimée parmi les intervalles [0, 10[, [10, 14[ et [14, 20]. Les pourcentages de ces intervalles seront comparés avec ceux des intervalles de notes effectives après le passage du test.

\section{Premières constatations}

Nous avons fait deux premières constatations concernant, d'une part, l'apprentissage en ligne et, d'autre part, l'autorégulation de l'apprenant, que nous avons présentées dans Daouas, Tounsi et Trigano (2007).

Les étudiants de $\mathrm{E}_{\mathrm{Net}}$ ont consulté NF01 à $75 \%$ et ceux de $\mathrm{E}_{\mathrm{Net}}$ ${ }_{\text {G3A' }}$ à 62,9\%. La totalité de ceux qui n'ont pas consulté le site ont répondu avoir l'intention de le faire. Ainsi, nous avons 
distingué trois types de motivation pour la consultation de NF01 et les mesures sont de 33,3\% pour s'améliorer, de 5,6\% parce que c'est imposé et de 61,1\% par curiosité.

Les étudiants de $\mathrm{E}_{\text {Net-G3A }}$ ont consulté le guide G3A à 27,3\%. Parmi ceux qui n'ont pas consulté, $76 \%$ ont répondu avoir l'intention de le faire et leur motivation est de 19,2 \% pour s'améliorer, de $0 \%$ parce que c'est imposé et de $80,8 \%$ par curiosité.

Comme le pourcentage des étudiants qui ont préféré consulter le site en ligne (75\%) est beaucoup plus élevé que celui des étudiants qui ont préféré lire le guide en papier que nous leur avons distribué $(27,3 \%)$, cela nous a amené à penser que l'une des hypothèses possibles est que les étudiants ont profité des avantages de l'apprentissage en ligne, à savoir la libération par rapport au lieu et au temps. résolution des exercices pour le lien «Exercices », la réponse au QCM pour le lien «QCM », l'exécution des simulations et jeux pour les liens «Simulations » et « Jeu » et, enfin, le téléchargement des parties du cours pour le lien « Téléchargements ». Un lien préféré est un lien qui a plu à l'étudiant qui l'a visité.

En répondant au questionnaire, l'étudiant devait cocher, parmi les types de liens, ceux qu'il a seulement visités, ceux qu'il a manipulés et ceux qu'il a préférés. Nous avons constaté que c'était plus facile de procéder à un recensement manuel des réponses, car chaque étudiant avait la possibilité de cocher
Nous avons également constaté que la motivation des étudiants pour la consultation du site en ligne et du guide est liée essentiellement à leur curiosité (61,1 \% pour NF01 et 80,8\% pour G3A) et non pas au fait qu'elle est imposée par le système du cours. Cela nous a amené à suggérer que les étudiants ont une tendance à devenir plus autonomes et autorégulés.

\section{Résultats par rapport au cours en ligne}

En premier lieu, nous avons fait une distinction entre les liens du cours visités, les liens manipulés et les liens préférés par les étudiants.

Un lien visité est un lien sur lequel un étudiant a cliqué sans trop s'attarder, passant rapidement à un autre lien. Un lien manipulé est un lien sur lequel un étudiant a cliqué, utilisant sa page correspondante pour effectuer des activités telles que lecture du texte du cours pour le lien «Théorique », la plusieurs liens simultanément.

Le Tableau 1 contient les pourcentages mesurés des étudiants ayant coché les différents liens qu'ils ont visités, manipulés et préférés, montrant le comportement des étudiants vis-à-vis des différents liens du site en ligne. Nous remarquons que les pourcentages les plus élevés sont ceux des liens «Théorique » et «Exercices ». Cela laisse penser que les étudiants sont très liés à leur cours en classe et qu'en consultant le site, ils ont cherché une certaine ressemblance avec ce dernier. Par conséquent, leur offrir les liens de «Simulations » et « Jeux pédagogiques » présente un changement pour eux par rapport à leur apprentissage habituel.

Néanmoins, bien que les liens « Simulations », «QCM » et « Jeu » représentent des pourcentages moins élevés, nous pouvons remarquer que la plupart des étudiants qui ont visité ces liens les ont appréciés, même s'ils ne les ont pas 
manipulés. En effet, sur les 21,05\% qui ont visité le lien «Simulations », 15,78 \% l'ont apprécié. En plus, tous les étudiants qui ont visité les liens «QCM » et «Jeu » les ont trouvés plaisants. Cela laisse penser que si les étudiants se trouvent motivés dans le cadre de leur cours à visiter tous ces liens, ils pourraient les apprécier à leur juste valeur. D'autre part, le lien « Téléchargements » a été visité par $15,78 \%$ des étudiants, mais n'a plu à personne. Ce lien permet de garder une copie du cours théorique; or les étudiants préféreraient celle du cours de leur enseignant, pensant que le test serait basé sur ce dernier plus que sur le cours en ligne.

Par ailleurs, nous nous sommes intéressé à l'avis des étudiants par rapport aux différents liens. Ainsi, sur une échelle allant de 1 à 5 , où 1 représente « trop peu » et 5 « énormé- explorer davantage le reste du site, qu'ils leur apportent un plus par rapport à leur cours en classe et qu'ils pourraient les aider dans leur test de contrôle. Cela confirme la constatation trouvée à partir du Tableau 1. Le lien «QCM » représente des pourcentages très proches avec des échelles 3 et 4 , les questions à choix multiples pouvant refléter le cours de l'enseignant où les étudiants doivent choisir les réponses exactes à partir d'affirmations ou de définitions.

Le lien «Simulations » représente 7,5\% à égalité pour les échelles 2 et 4 . Visiblement, les étudiants ne sont pas tous d'accord sur le fait que ce lien motive « un peu » ou « beaucoup » à explorer le reste du site; c'est un outil pédagogique tout à fait nouveau pour eux. Par contre, leur avis se précise plus pour les deux questions suivantes : ils sont d'avis à $10 \%$ seulement que ce lien apporte une plus-value

\begin{tabular}{|c|c|c|c|c|c|c|c|}
\hline & & Théorique & Exercices & Simulations & $\mathrm{QCM}$ & Jeu & Téléchargements \\
\hline \multirow{2}{*}{$\begin{array}{l}\text { Motivation à explorer } \\
\text { plus le site }\end{array}$} & $\%$ & 27,5 & 17,5 & 7,5 & 12,5 & 10 & 12,5 \\
\hline & Échelle & 3 & 3 & 2 et 4 & 3 & 4 & 1 \\
\hline \multirow[t]{2}{*}{ Plus-value apportée } & $\%$ & 15 & 15 & 10 & 15 & 10 & 10 \\
\hline & Échelle & 3 & 2 & 2 & 3 & 1 et 2 & 1 et 2 \\
\hline \multirow[t]{2}{*}{ Aide pour le test de contrôle } & $\%$ & 22,5 & 17,5 & 10 & 10 & 17,5 & 12,5 \\
\hline & Échelle & 3 & 4 & 1 & 4 & 1 & 1 et 2 \\
\hline
\end{tabular}

ment », et pour chaque lien, nous avons demandé aux étudiants à quel point ces sections les ont motivés pour explorer encore plus le site, à quel point ces parties ont constitué un plus par rapport à leur cours et à quel point ces parties pourraient les aider dans leur test de contrôle. Nous avons relevé les pourcentages des échelles cochées les plus élevées pour chaque lien dans le Tableau 2.

La première remarque générale, d'après le Tableau 2, est que les liens «Théorique » et «Exercices » représentent les pourcentages les plus élevés avec une échelle moyenne de 3 pour la plupart des réponses. La majorité des étudiants seraient donc d'avis que ces liens les motivent pour et aide pour le test de contrôle. Effectivement, lors du test, les étudiants auront du mal à imaginer l'application des simulations du site dans leurs réponses.

Le lien «Jeu » motive beaucoup les étudiants pour explorer le reste du site, mais nous remarquons qu'il est assez difficile pour eux d'évaluer la plus-value qu'apportent les jeux pédagogiques et comment ils peuvent les aider dans la réussite du test puisque les échelles utilisées sont «trop peu » et « un peu », soit 1 et 2 .

Pour finir, le lien «Téléchargements » reste celui qui motive le moins les étudiants. Ils ne sont pas intéressés par le fait de garder une copie des parties du cours. Ils préféreraient 
réviser à partir du cours de leur enseignant pour leur test de contrôle pour mieux garantir leurs notes.

Dans ce contexte d'apprentissage en ligne, nous avons voulu connaître le pourcentage des étudiants ayant suivi, auparavant, un cours en ligne. De même, nous avons voulu estimer leurs motivations pour refaire l'expérience. Pour la majorité des étudiants des deux échantillons $E_{\mathrm{Net}}$ et $\mathrm{E}_{\mathrm{Net-G3A^{ \prime }}}$ à savoir $70 \%$, ce contact avec un site en ligne représente une première expérience de ce genre. On ne tient pas compte de l'échantillon $E_{0}$ puisqu'il n'a pas eu accès au site en ligne. En outre, les pourcentages les plus élevés sont de $25 \%$ pour les étudiants affirmant qu'ils sont tout à fait motivés pour refaire l'expérience dans d'autres domaines et de $32,5 \%$ pour ceux

Tableau 3. Caractéristiques de l'autorégulation chez les étudiants
\begin{tabular}{|l|c|c|}
\hline Caractéristiques de l'autorégulation & Plus grand pourcentage & Échelle \\
\hline Se fixer des objectifs réalisables & 32,5 & 3 \\
\hline Motivation pour volonté de maîtrise & 40 & 4 \\
\hline Planifier et gérer son temps & 30 & 3 \\
\hline Avoir des stratégies sociales & 30 & 3 \\
\hline Transposer les exemples à sa propre expérience & 30 & 3 \\
\hline Avoir des stratégies cognitives & 32,5 & 2 \\
\hline S'autocontrôler & 30 & 2 \\
\hline
\end{tabular}

qui affirment que ce mode d'apprentissage serait tout à fait utile dans leur vie professionnelle.

Les réponses relevées montrent que les étudiants sont très liés au cours de leur enseignant. Il serait intéressant de refaire l'expérimentation dans un autre contexte où les étudiants disposeront seulement du cours en ligne pour comparer leurs réponses et de voir si elles changeraient par rapport à celles-ci.

\section{Résultats par rapport au guide G3A}

Seul l'échantillon $E_{\text {Net-G3A }}$ est concerné par cette section du questionnaire. Les étudiants de cet échantillon devaient répondre aux questions en utilisant une échelle de 1 à 5, où 1 représente «Pas du tout » et 5 « Tout à fait ». Nous avons relevé les pourcentages des échelles cochées les plus élevées dans le Tableau 3 pour avoir une idée générale de l'autorégulation des étudiants.

La moyenne des pourcentages du tableau représente $32 \%$ avec des échelles entre 2 et 4 . Ces pourcentages représentent des étudiants qui pensent (échelle 3) qu'ils sont moyennement capables de se fixer des objectifs réalisables, qui sont très motivés (échelle 4) pour mieux maîtriser le cours, qui pensent moyennement (échelle 3) pouvoir gérer leurs temps, qui peuvent moyennement (échelle 3) avoir des stratégies sociales dans leurs apprentissages, qui peuvent moyennement (échelle 3) transposer les exemples du guide à leurs propres expériences et qui peuvent faiblement (échelle 2) avoir des stratégies cognitives et s'autocontrôler.

Le fait que les valeurs des pourcentages ne représentent pas de grandes différences les unes par rapport aux autres indique que l'échantillon est assez homogène. Par contre, les échelles choisies reflètent une attitude qui n'est pas très sûre, voire indifférente, ce qui indique que les étudiants manquent encore de confiance et doivent apprendre à être encore plus autorégulés.

Il serait intéressant d'améliorer le guide en réalisant un Site Interactif pour 1'Apprenant Autonome qui remplacerait le guide en papier dans l'expérimentation et motiverait mieux les étudiants à améliorer leur autonomie et leur autorégulation.

\section{Résultats après le test}

Il est utile de rappeler qu'à travers la comparaison des notes des étudiants, notre objectif n'est pas de vérifier si ces notes se sont 
améliorées d'un échantillon à un autre, mais plutôt de détecter certaines caractéristiques chez les étudiants qui pourraient nous donner une indication sur leur autorégulation. Dans le test donné par l'enseignant commun aux trois échantillons, les étudiants sont tenus de résoudre trois exercices présentés sous forme d'énoncés et nécessitant l'écriture de trois algorithmes. En outre, la notation adoptée par les enseignants dans tous les cours est la notation sur 20, où 10 représente une note moyenne. Nous avons utilisé trois intervalles pour relever les pourcentages des notes des étudiants, [0, 10[, représentant les étudiants qui sont inférieurs à la moyenne, [10, 14[, représentant les étudiants moyens et [14, 20], représentant les bons étudiants.

\section{Auto-estimation}

Les recherches ont montré que l'incapacité de juger ses propres habiletés conduit à un échec de l'autorégulation (Famose, 2003). En comparant les pourcentages estimés des différents intervalles, nous remarquons que pour $\mathrm{E}_{0}$, le pourcentage estimé le plus élevé est de 57,1 \% pour l'intervalle $[10,14[$, alors que le pourcentage effectif le plus élevé est de 58,4 \% pour l'intervalle [0, 10[. Cela montre une faible capacité d'auto-estimer son intervalle de note chez la majorité des étudiants de cet échantillon. Nous rappelons que les étudiants de $\mathrm{E}_{0}$ ont suivi le cours classique sans accès au site en ligne ni au guide G3A, et qu'ils n'ont donc eu aucune aide pour améliorer leur autorégulation.

Tableau 4. Pourcentages des notes estimées et effectives selon les trois échantillons
\begin{tabular}{|l|l|l|l|l|}
\hline Intervalles de notes & & {$[\mathbf{0 , 1 0}[$} & {$[\mathbf{1 0}, \mathbf{1 4}[$} & {$[\mathbf{1 4 , 2 0 ]}$} \\
\hline \multirow{2}{*}{$\mathrm{E}_{0}$} & Estimés & 28,5 & 57,1 & 14,4 \\
\cline { 2 - 6 } & Effectifs & 58,4 & 16,6 & 25 \\
\hline \multirow{2}{*}{$\mathrm{E}_{\mathrm{Net}}$} & Estimés & 50 & 33,4 & 16,6 \\
\cline { 2 - 6 } & Effectifs & 70 & 20 & 10 \\
\hline \multirow{2}{*}{$\mathrm{E}_{\text {Net-G3A }}$} & Estimés & 50 & 41,7 & 8,3 \\
\cline { 2 - 6 } & Effectifs & 80 & 10 & 10 \\
\hline
\end{tabular}

Quant à $\mathrm{E}_{\mathrm{Net}}$ et $\mathrm{E}_{\mathrm{Net-G3A}}$ les pourcentages estimés les plus élevés sont de $50 \%$ pour l'intervalle $[0,10[$, alors que les pourcentages effectifs les plus élevés sont de $70 \%$ pour $\mathrm{E}_{\mathrm{Net}}$ et de $80 \%$ pour $\mathrm{E}_{\mathrm{Net-G3A}}$ pour le même intervalle [0, 10[. Cela montre que la majorité des étudiants de $\mathrm{E}_{\mathrm{Net}}$ et de $\mathrm{E}_{\mathrm{Net}-\mathrm{G} 3 \mathrm{~A}}$ sont capables d'auto-estimer leur intervalle de note, étant ainsi plus

Le Tableau 4 présente les pourcentages des notes estimées et effectives des trois échantillons. En calculant ces pourcentages, nous étions intéressé par les trois types de comparaisons suivantes :

- La première est la comparaison des pourcentages estimés et des pourcentages effectifs pour les trois échantillons; cela nous permettra d'avoir une indication sur la capacité des étudiants à auto-estimer leur intervalle de note.

- La deuxième est la comparaison des pourcentages estimés des différents intervalles des trois échantillons; cela nous permettra d'avoir une indication sur le degré d'estime de soi des étudiants.

- La troisième est la comparaison des pourcentages effectifs des différents intervalles des trois échantillons; cela nous permettra de voir si, par rapport aux notes de $\mathrm{E}_{0}$ et $\mathrm{E}_{\mathrm{Net}^{\prime}}$ il existe une influence à la suite de l'utilisation du cours en ligne et si, par rapport aux notes de $\mathrm{E}_{\mathrm{Net}}$ et $\mathrm{E}_{\mathrm{Net}^{-G 3 \mathrm{~A}^{\prime}}}$ il existe une influence à la suite de l'utilisation du guide. aptes à devenir autorégulés dans leur apprentissage.

\section{Estime de soi}

D'après Famose (2003), une basse estime de soi conduit l'apprenant à se fixer des buts peu avantageux comportant moins de défis afin de fuir l'échec. En revanche, une haute estime de soi conduit l'apprenant à affronter des situations qui posent plus de défis, par orgueil et par fierté. Par conséquent, une haute estime de soi peut être avantageuse pour l'autorégulation, compte tenu des défis à relever. Néanmoins, elle peut avoir parfois des conséquences néfastes. Ce type d'apprenant n'acceptant pas l'échec par orgueil et par fierté, il peut rencontrer des situations de blocage qui le conduisent à un échec.

En comparant les pourcentages des intervalles estimés, nous remarquons que la majorité des étudiants de $\mathrm{E}_{0}(57,1 \%)$ pensent avoir leurs notes dans l'intervalle [10, 14[. Cela représente une estime de soi moyenne. Par contre, cette estime 
est moins élevée pour la majorité des étudiants de $\mathrm{E}_{\mathrm{Net}^{\prime}} 50 \%$ estimant leurs notes dans l'intervalle [0, 10[. Pour $\mathrm{E}_{\text {Net-G3A' }^{\prime}}$ l'estime de soi remonte par rapport à celle des étudiants de $\mathrm{E}_{\mathrm{Net}}$. En effet, la majorité des étudiants, $50 \%$ et $41,7 \%$, estiment leurs notes dans les intervalles [0, 10[ et [10, 14[, respectivement. Par conséquent, nous pouvons remarquer que le guide semble avoir contribué à l'amélioration de l'estime de soi des étudiants.

\section{Influence du cours en ligne et du guide}

En examinant les pourcentages de notes effectives des étudiants dans $\mathrm{T} 4$, nous remarquons que ceux de $\mathrm{E}_{0}$ semblent bien répartis entre les intervalles. Les notes sont plus faibles pour les étudiants de $\mathrm{E}_{\mathrm{Net}}$ et s'affaiblissent encore pour $\mathrm{E}_{\mathrm{Net-G3A}}$. Cela est dû, d'une part, à la nature du test qui est lié d'une façon directe au cours de l'enseignant en classe et, d'autre part, aux étudiants qui n'ont pas su exploiter le site en ligne pour améliorer leur niveau de maîtrise du cours et n'ont pas pu s'inspirer des propositions et directives du guide pour trouver leurs propres stratégies d'apprentissage et de préparation du test. Par conséquent, il serait recommandé de changer le système des cours utilisé avec les étudiants pour leur enseignement et leur évaluation. Il faudrait employer des méthodes qui contribuent à favoriser une plus grande autonomie de l'étudiant et à le motiver à diversifier ses ressources d'apprentissage.

\section{Conclusion}

Nous avons remarqué, à la suite de cette expérimentation, que les étudiants sont encore trop attachés à leur cours en classe, ce qui affecte leur degré d'autorégulation et d'autonomie. D'autre part, les étudiants préfèrent la consultation en ligne à la lecture sur papier. Par ailleurs, nous avons remarqué une amélioration de l'auto-estimation des étudiants ainsi que de leur estime de soi. Par contre, ils n'ont pas été capables d'utiliser les bonnes stratégies d'apprentissage pour améliorer leurs notes.

Dans une prochaine étude, nous envisageons de refaire l'expérimentation avec la réalisation d'un site interactif pour l'ap- prentissage autorégulé, remplaçant le guide G3A en papier, pour inciter et motiver davantage les étudiants à améliorer leur autorégulation et à mieux l'adapter à l'apprentissage en ligne.

\section{Références}

Abdelli, Z. (avec Nyahoho, E. et Marleau, M.). (2003). Formation en ligne et PME québécoises - Occasions et perspectives. Montréal : Direction du développement des entreprises et des affaires du ministère du Développement économique et régional du Québec.

Alexander, P. A., Graham, S. et Harris, K. R. (1998). A perspective on strategy research: Progress and prospects. Educational Psychology Review, 10(2), 129-154.

Ames, C. (1992). Classrooms: Goals, structures, and student motivation. Journal of Educational Psychology, 84(3), 261-271.

Anderman, E. M. et Maehr, M. L. (1994). Motivation and schooling in the middle grades. Review of Educational Research, 64(2), 287-309.

Balancier, P., Georges, F., Jacobs, S., Martin, V. et Poumay, M. (2006). L'e-learning dans l'enseignement supérieur - Environnement international francophone (Rapport de synthèse). Liège, Belgique : Université de Liège, Laboratoire de Soutien à l'Enseignement Télématique (LabSET).

Daouas, T., Tounsi, M. et Trigano, P. (2007, mai). Apprentissage en ligne autorégulé. Communication présentée au 4 e Symposium International en EAD, Hammam Sousse, Tunisie.

Deci, E. L. et Ryan, R. M. (2000). The what and why of goal pursuits: Human needs and the self-determination of behavior. Psychological Inquiry, 11(4), 227-268.

Famose, J.-P. (2003). Réflexion sur les programmes : à propos de l'apprentissage autorégulé. Qu'est-ce qu'apprendre en éducation physique et sportive? Revue EP.S, 300, 13-20.

Hrimech, M. (2000). Les stratégies d'apprentissage en contexte d'autoformation. Dans R. FaucheretM.Hrimech(dir.), L'autoformation dans l'enseignement supérieur : apports européens et nord-américains pour l'an 2000 (p. 99-111). Montréal : Les éditions Nouvelles.

Lapan, R. T., Cardash, C. M. et Turner, S. (2002). Empowering students to become self-regulated learners. Professional School Counseling, 5(4), 257-265.

Lebow, D. (1993). Constructivist values for instructional systems design: Five principles toward a new mindset. Educational Technology Research and Development, 41(3), 4-16. 
Lucas, A. F. (1990). Using Psychological Models to Understand Student

Motivation. Dans M. D. Svinicki (ed.), The Changing Face of College Teaching. New Directions for Teaching and Learning, no. 42. San Francisco: Jossey-Bass.

Mayer, R. E. (1996). Learning strategies for making sense out of expository text: The SOI model for guiding three cognitive processes in knowledge construction. Educational Psychology Review, 8(4), 357-372.

McCormick, C. B. et Pressley, M.(1997). Educational psychology: Learning, instruction, and assessment. New York : Longman.

Organisation de coopération et développement économiques [OCDE]. (2004). Apprendre aujourd'hui, réussir demain - Premiers résultats de PISA 2003. Paris : auteur.

Paris, S. G. et Winograd, P. (1997). The role of self-regulated learning in contextual teaching: Principles and practices for teacher preparation (rapport préparé pour le US Department of Education, Office of Educational Research and Improvement). (ERIC Document Reproduction Service No. ED479905)

Pintrich, P. R. (2000). The role of goal orientation in self-regulated learning. Dans M. Boekaerts, P. R. Pintrich et M. Zeidner (dir.), Handbook of selfregulation (p. 452-502). San Diego, CA : Academic Press.

Pintrich, P. R. et Schunk, D. H. (1996). Motivation in education: Theory, research and applications. Englewood Cliffs, NJ : Prentice Hall Merrill.

Roadmap to communicating knowledge essential for the indusTrial environment [ROCKET] consortium (WP2 partners). (2003). The stateof-the-art of e-Learning (Deliverable D 2.2). Florence, Italie : auteur.

Ruph, F. et Hrimech, M. (2001). Les effets perçus d'un atelier d'efficience cognitive sur le changement de stratégies d'apprentissage d'étudiants universitaires. Revue des sciences de l'éducation, 27(3), 595-620.

Svinicki, M. D. (2004, octobre). Learning, motivation and student selfregulation. Communication présentée au Dane and Mary Louise Miller Symposium and CASEE Annual Meeting, Savannah, GA.

Trigano, P. (2006). Self-regulated learning in a TELE at the Université de Technologie de Compiègne: An analysis from multiple perspectives. European Journal of Education, 41(3-4), 381-395.

Wade, S. E., Trathern, W. et Schaw, G. (1990). An analysis of spontaneous study strategies. Reading Research Quarterly, 25(2), 147-166.

Weinstein, C. E. (1988). Assessment and training of student learning strategies. Dans R. R. Schmeck (dir.), Learning strategies and learning styles (p. 291-316). New York : Plenum Press.
Wilson, J. (1997, juin). Self-regulated learners and distance education theory. Récupéré du site du College of Education de l'Université de Saskatchewan, section Educational Links and Ressources - Occasional Papers : http: / / www.usask.ca/education/ coursework/802papers /

Zimmerman, B. J. (2002). Becoming a self-regulated learner: An overview. Theory Into Practice, 41(2), 64-72.

Zimmerman, B. J., Bonner, S. et Kovach, R. (1996). Developing selfregulated learners: Beyond achievement to self-efficacy. Washington, DC : American Psychological Association.

\section{Notes}

1 Les idées de travaux à effectuer, à la suite de ce travail, représenteront une partie d'un projet de recherche qui regroupera quatre universités partenaires, à savoir l'Université de Technologie de Compiègne en France, l’Université de Craiova en Roumanie, l'Institut National d'Informatique en Algérie et l'Institut des Hautes Études Commerciales de Carthage en Tunisie.

2 Des remerciements, dans le cadre de ce travail, sont à présenter au Pr Philippe Trigano, de l'Université de Technologie de Compiègne, qui nous a donné l'accès au cours en ligne NF01 dont il est responsable, ainsi qu'à l'étudiant au Master Marouene Tounsi, qui a contribué aux expérimentations.

3 Les lecteurs intéressés par la publication des premiers résultats de cette expérimentation peuvent contacter l'auteur à son adresse électronique.

\section{Annexe 1 (en ligne)}

Questionnaire sur l'apprentissage en ligne autorégulé

http:/ / www.profetic.org/ revue/ IMG / pdf/ ritpu0401

questionnaire_daouas.pdf 